\title{
Zärtlichkeit und Offenheit
}

\author{
Möglichkeiten, das Alter in Wohlbefinden zu genießen
}

Einsamkeit tritt im Alter nicht häufiger auf als in der Jugend. Die Alterseinsamkeit wird jedoch in den Medien überrepräsentiert. Das ist nur eine Erkenntnis, die kürzlich bei der Fachtagung „Einsamkeit im Alter" des privaten Pflegeheimbetreibers SeneCura diskutiert wurde. Ältere Menschen sind jedoch aufgrund mancher körperlicher und kognitiver Einschränkungen eher in ihrer Bewegungsfreiheit beeinträchtigt. Gerade hier können Medien dazu beitragen, die Alterseinsamkeit zu überwinden.

Diskriminierende Bilder von älteren Menschen prägen das verbreitet negative $\mathrm{Al}-$ ternsbild, stellte Dr. Ulla Kriebernegg, Zentrum für Inter-Amerikanische Studien an der Universität Graz, fest. Dagegen sollten verstärkt Beispiele von „comfortable aging" aufgezeigt werden. Als ein schillerndes Beispiel dafür gab Publikumsliebling Otto Schenk Einblicke in seinen Alltag des älter Werdens. Mit 85 Jahren werden körperliche Beeinträchtigungen zwar zunehmend spürbar, die Gefühlswelt behält ihren Stellenwert bei oder wird sogar wichtiger. Der Wunsch älterer Menschen nach Zärtlichkeit wird oft viel zu wenig wahrgenommen. Im Alter, so der Schauspieler und Regisseur, „eröffnen sich neue Gebiete. Das Gebiet der Zärtlichkeit zum Beispiel wird unbeschreiblich wichtig im Alter, jeder Handgriff, den man macht und jede Berührung mit einem Menschen, den man gern hat, kriegt etwas Zauberhaftes, etwas Magisches." Und er plädiert für den Humor: „Das Lachen darf man nie verlernen. Das Lachen ist das, was uns vom Affen unterscheidet. Fast das einzige. Politisch sind sie uns weit überlegen. Und suchen Sie jede Möglichkeit, einem alten Menschen ein Lachen abzugewinnen", forderte er die Mitarbeiter der Gesundheits- und Krankenpflege auf: „Schon wenn er lächelt, schaut er um zehn Jahre jünger aus. Auch zahnlos.“

\section{Lernen findet in jedem Dialog statt}

Offen zu sein für Neues und für die Auseinandersetzung mit jüngeren Menschen ist nicht nur für Schenk sondern auch für den Soziologen Univ.-Prof. Dr. Franz Kolland, Institut für Soziologie an der Universität

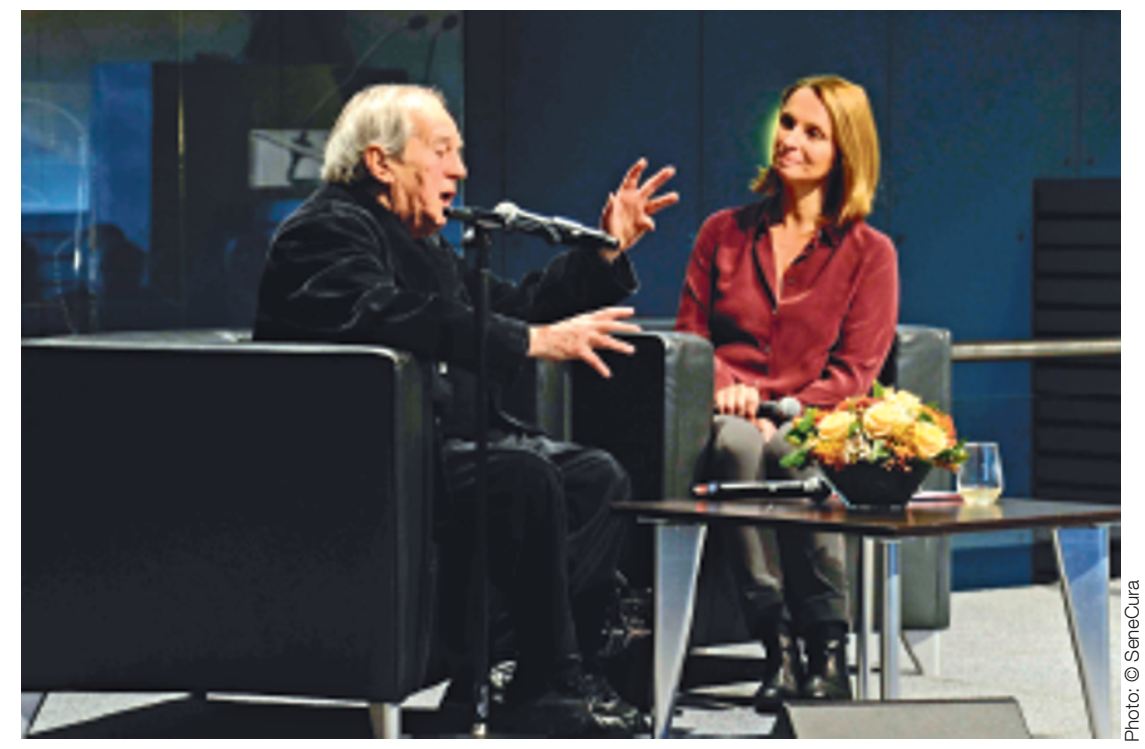

Otto Schenk: „Das Lachen darf man nie verlernen!“

Wien, ein Schlüssel zum Wohlbefinden und für den möglichst langen Erhalt der kognitiven Fähigkeiten. Dabei gehe es nicht nur um lebenslanges Lernen, sondern vor allem auch um das Lernen für ein langes Leben. „Lernen hilft uns“, so Kolland, „unsere Veränderungsbereitschaft zu wahren und unsere Anpassungsfähigkeit zu verbessern. Unter Lernen im Alter verstehen wir aber nicht nur normale Bildungsprozesse. Vielmehr geht es um ein dialogisches Lernen im aktiven Austausch mit anderen Menschen - egal ob dies Lehrende oder gute Freunde sind, mit denen man sich über ein Thema regelmäßig austauscht.“

Ebenfalls eine Form des Austauschs und der emotionalen und sozialen Anregung ist der Kontakt mit Tieren. Wissenschaftliche Erkenntnisse legen nahe, dass ältere Menschen vom Kontakt mit Tieren genauso profitieren wie Kinder. „Tierkontakt fördert die emotionale, soziale, physische und kognitive Fitness von älteren Menschen“, berichtete Univ.-Prof. Kurt Kotrschal, Leiter des Instituts für interdisziplinäre Forschung der Mensch-Tier Beziehung. „Tiere können dank der Stimmungsübertragung zum Beispiel beruhigend auf Menschen wirken, oder aber auch aktivieren oder positive Emotionen wie Freude oder Zuneigung auslösen. Sie sind deshalb wertvolle ,Unterstützer ‘ sowohl im privaten Bereich als auch bei
Therapien“. Eine möglichst naturnahe Lebensraumgestaltung und die Ermöglichung von Tierhaltung in Pflegeinstitutionen könnten diese Beziehung aktiv fördern.

\section{Moderne Medien verbinden Zeit und Raum}

Die zunehmend interaktiv gestaltbaren modernen Medien können nicht nur als passiver Zeitvertreib genutzt werden, sondern sind heute bereits Beitrag zu gesellschaftlicher Teilhabe. Teilweise Schwellenängste älterer Menschen vor der modernen Technik können durch entsprechende Begleitung überwunden werden. Kinder und Enkelkinder oder Kooperationen mit Schulen stellen einen wichtigen Beitrag in mehrfacher Hinsicht dar: Gegenseitige Wertschätzung und Kommunikation werden in einem Lernprozess ermöglicht. Fernsehen eröffnet aber auch eine Möglichkeit der Auseinandersetzung und Bewältigung von Vergangenheit, Gegenwart und Zukunft, und kann der biographischen Selbst-und Weltreflexion dienen, stellte Univ.-Prof. Dr. Anja Hartung, Vorsitzende des Vereins Gesellschaft - Altern - Medien, fest. Offenheit ist auch hier gefragt.

Quelle: Presseaussendung SeneCura, SeneCura Forum „Einsamkeit im Alter“, November 2015, Wien 